Bull. Austral. Math. Soc.

VOL. 54 (1996) [167-176]

\title{
AN ALGORITHM FOR CONSTRUCTING LOCALLY OPTIMAL MIN-MAX TRIANGULATION
}

\author{
M. Shrivastava
}

\begin{abstract}
Several interesting criteria for constructing triangulations associated with a given set of points in a plane have been introduced. In order to obtain optimal triangulation with respect to the min-max-angle criterion, it is essential to study the nature of neutral cases with respect to the criterion. Our aim in this paper is to establish precise equations for neutral set curves with respect to the min-max-angle criterion and to develop an algorithm to obtain a locally optimal triangulation with respect to the criterion.
\end{abstract}

\section{INTRODUCTION}

Triangulations play a very significant role in problems of interpolation and approximation of bivariate functions. Bivariate splines which are certain piecewise polynomials defined over triangulations of domains in $\mathbb{R}^{2}$ have proved to be useful tools in approximation processes, finite element-methods, computer aided geometric designs and other such fields. Lawson [4] and Schumaker [5] have designed several interesting criteria for constructing triangulations of the convex hull of a given set of points in a plane. Most popular choices for triangulation criteria are the max-min-angle criterion and the minmax angle criterion. The max-min-angle criterion is quite well examined and efficient algorithms have been developed for finding optimal triangulations with respect to this criterion (see Correc and Chapius [1], Field [2], Lawson [4] and others). Considering the min-max triangulation criterion, Hansford [3] has demonstrated the graphic construction of neutral sets of given three points. In the present paper we aim to obtain precise equations for the neutral set curves with respect to the min-max angle criterion. Moreover we design a practical algorithm for finding a locally optimal triangulation with respect to this criterion.

\section{Prefatory CONDITIONS}

Let $D=\left\{\left(x_{i}, y_{i}, f_{i}\right): x_{i}, y_{i} \in \mathbb{R}\right\}_{i=1}^{n}$ be a set of scattered data where $V=\left\{v_{i}=\right.$ $\left.\left(x_{i}, y_{i}\right): x_{i}, y_{i} \in \mathbb{R}\right\}_{i=1}^{n}$ is a set of $n$ distinct points in the plane. Let $\Omega$ be the convex

Received 23 October 1995

The author would like to thank Dr. K.C. Deomurari for some helpful discussions.

Copyright Clearance Centre, Inc. Serial-fee code: 0004-9729/96 \$A2.00+0.00. 
hull of $V$. In order to obtain a surface model to the data $D$ in $\Omega$, we triangulate the region $\Omega$. Given a set $V$ of vertices, $\Omega$ can be triangulated in several ways. Different triangulation criteria enable us to choose a triangulation suitable for the practical needs of our problem. A triangulation criterion chooses the preferred triangulation among several alternatives, thus defining an ordering on the set of all triangulations. We use the notation $T^{\prime}<T$ to denote that the criterion prefers $T^{\prime}$ to $T$.

An optimal triangulation of $\Omega$ with respect to given criterion is a triangulation $T^{\prime}$ such that $T^{\prime}<T$ for every triangulation $T$ of $\Omega$. An optimal triangulation of $\Omega$ always exists since there are only a finitely many triangulations of $\Omega$, yet it might be difficult to attain this optimal triangulation. Thus, in general, we try to get a locally optimal triangulation. Let $T$ be a triangulation of $\Omega, e$ an internal edge of $T$, and $Q$ a quadrilateral formed from the two triangles having $e$ as a common edge. Then edge $e$ is called locally optimal if one of the following conditions holds:

1. The quadrilateral $Q$ is not strictly convex:

2. The quadrilateral $Q$ is strictly convex and $T<T^{\prime}$ where $T^{\prime}$ is obtained from $T$ by of replacing $e$ by the other diagonal of $Q$.

A locally optimal triangulation of $Q$ is a triangulation in which all edges are locally optimal.

Definition 2.1: Min-max-angle criterion. Given a triangle $T$, let $\alpha(T)$ $=$ (maximum angle in $T$ ). Corresponding to a triangulation $\Gamma$, let $\alpha(\Gamma)=\max$ $\{\alpha(T): T \in \Gamma\}$. Then $\Gamma$ is said to be better than triangulation $\Gamma^{\prime}$ with respect to the min-max-angle cirterion provided that $\alpha(\Gamma) \leqslant \alpha\left(\Gamma^{\prime}\right)$.
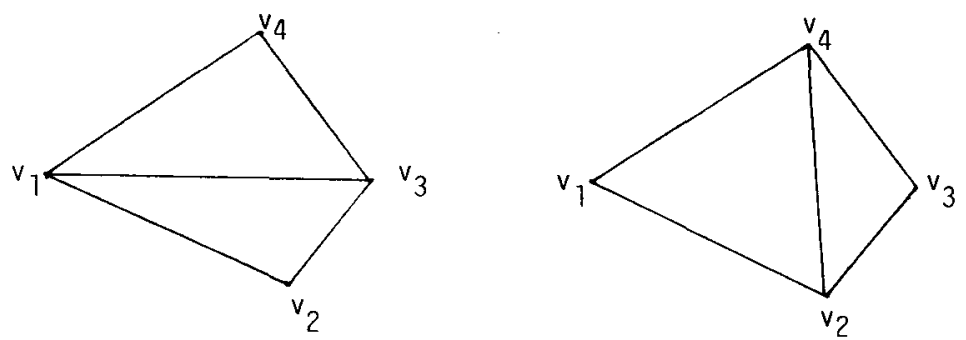

Figure 2.1

Let $v_{1}, v_{2}, v_{3}, v_{4}$ be four given points. Then there are two diagonals and correspondingly two triangulations as shown in Figure 2.1.

A triangulation criterion determines which diagonal should be used to obtain a better triangulation. A neutral case with respect to a triangulation-criterion occurs in a convex quadrilateral when either of the two diagonals may be chosen. Given three 
points, if a fourth point is determined so that a convex quadrilateral with the neutral case is formed then the locus of such a fourth point is called the neutral set for the given three points. In the case of the max-min-angle criterion, the neutral set is the circum circle of the given three points. However, in the case of the min-max-angle criterion the determination of the neutral set is slightly more involved. We shall obtain the precise equations to the curve/pieces of curves of the neutral sets corresponding to all possible relative positions of the given three points.

\section{The NeUtral SET For THE MiN-MAX-ANGLE CRITERION}

Let $v_{1}, v_{2}$ and $v_{3}$ be three given points in anticlockwise order. In triangle $v_{1} v_{2} v_{3}$ let the measure of angles at vertices $v_{1}, v_{2}$ and $v_{3}$ be $A, B$ and $C$ respectively and let the sides opposite these angles be $l_{1}, l_{2}$ and $l_{3}$ respectively. Let $x$ be the position of a fourth point such that in the convex quadrilateral $v_{1} v_{2} v_{3} x$ the neutral case occurs with respect to the min-max-angle criterion. With $v_{1}$ as origin and $v_{1} v_{2}$ as initial line let $x$ be the point $(r, \theta)$ so that $v_{1} x=r$ and $\angle v_{2} v_{1} x=\theta$. The locus of point $x$ determines the neutral set for the given three points. The following lemma (see Hansford [3]) gives an important assertion about the neutral case for the min-max-angle criterion:

LEMMA 3.1. In order that the neutral case with respect to the min-max-angle criterion may occur in a convex quadrilateral, two adjacent angles in it must be equal and they must be the maximum angles in the quadrilateral.

We proceed to obtain the neutral sets in different situations of the given three points $v_{1} v_{2} v_{3}$.

CASE $1 . B \leqslant 90^{\circ}$ :

Here we consider the case when $\angle v_{1} v_{2} v_{3}=B \leqslant 90^{\circ}$ as shown in Figure 3.1.

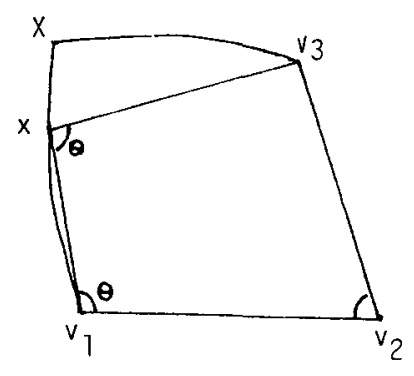

Figure 3.1

According to Lemma 3.1, in order that a neutral case may occur in the quadrilateral $v_{1} v_{2} v_{3} x$, two adjacent angles must be equal and must be the maximum angles in the 
quadrilateral. We first suppose the equal and maximum angles to be at vertices $v_{1}$ and $x$. Thus $\angle v_{2} v_{1} x=\theta=\angle v_{1} x v_{3}$. Obviously $\theta \geqslant 90^{\circ}$. Using the sine formula in triangle $x v_{1} v_{3}$ we easily find that

$$
r=l_{2} \frac{\sin (2 \theta-A)}{\sin \theta}
$$

This is a curve which starts from $v_{1}$ where $\theta$ is $90^{\circ}+A / 2$. When $x$ moves along the curve, the vectorial angle $\theta$ for $x$ decreases whereas $\angle v_{2} v_{3} x$ goes on increasing. Thus at a point $X$ where $\theta=120^{\circ}-B / 3$, we find that $\angle v_{2} v_{1} X=\angle v_{1} X v_{3}=\angle v_{2} v_{3} X$. At this point $X$, radius vector $r_{X}$ is given by

$$
r_{X}=l_{2} \frac{\sin \left(60^{\circ}+C+B / 3\right)}{\sin \left(60^{\circ}+B / 3\right)} .
$$

It is interesting to observe that if $\theta$ is decreased further, $\angle v_{2} v_{3} x$ exceeds $\angle v_{2} v_{1} x$ and we may have a neutral case for the quadrilateral only if the angle at $v_{3}$ is one of the maximum, adjacent and equal angles for the quadrilateral. Therefore now we suppose that the maximum and equal angles are at $v_{3}$ and $x$. Let $\bar{x}=(\bar{r}, \bar{\theta})$ be a position of $x$ in this situation. Therefore if $\angle v_{1} \bar{x} v_{3}=\angle v_{2} v_{3} \bar{x} \equiv \alpha$, then $2 \alpha=360^{\circ}-\bar{\theta}-B$. Hence from $\Delta v_{1} \bar{x} v_{3}$ we have

$$
\bar{r}=l_{2} \frac{\sin (\alpha-C)}{\sin \alpha} .
$$

Therefore the equation to this second part of the locus of point $x$ is given by

$$
r=l_{2} \frac{\sin (\theta / 2+B / 2+C)}{\sin (\theta / 2+B / 2)} .
$$

Obviously at $\theta=A$, we have $r=l_{2}$. Further we see that when $B=90^{\circ}, r_{X}=l_{1}$.

It is easy to see that at $\theta=120^{\circ}-B / 3$ the common values $r_{X}$ of the radius vector $r$ for the two parts of the locus of $x$ is given by (3.2). Thus we have proved the following:

THEOREM 3.1. Given three points $v_{1}, v_{2}$ and $v_{3}$ such that $\angle v_{1} v_{2} v_{3} \leqslant 90^{\circ}$, the neutral set with respect to the min-max angle criterion opposite to vertex $v_{2}$ is a continuous piecewise curve from $v_{1}$ to $v_{3}$ whose two parts $v_{1} X$ and $v_{3} X$ are given by equations (3.1) and (3.3) respectively.

REMARK 3.1. It is interesting to note that the curve constituting the neutral set has two pieces whose domains are given by $90^{\circ}+A / 2 \geqslant \theta \geqslant 120^{\circ}-B / 3$ and $120^{\circ}-B / 3 \geqslant \theta \geqslant A$ respectively. Therefore first part of the neutral set curve will disappear if $3 A+2 B \leqslant 180$ and the neutral set curve from $v_{1}$ to $v_{3}$ will be given by equation (3.3). Similarly neutral-set-curve will consist of a single curve given by (3.1), when $A+B / 3 \geqslant 120^{\circ}$. 


\section{Case 2: The neutral Cases When $B>90^{\circ}$}

Before we prove our main result for this case, we shall prove one proposition for the situation $90^{\circ}<B<120^{\circ}$.

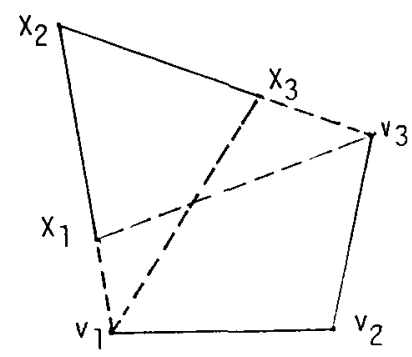

Figure 4.1

Let us suppose that $90^{\circ}<B<120^{\circ}$ and adjacent angles $\angle v_{1} v_{2} v_{3}$ and $\angle v_{2} v_{1} x$ are the maximum and equal angles of the convex quadrilateral $v_{1} v_{2} v_{3} x$. We observe that the neutral case for the quadrilateral occurs till $\angle v_{1} x v_{3} \leqslant B$ and $\angle v_{2} v_{3} x \leqslant B$. Clearly when $\angle v_{2} v_{3} x<B$, and the equal maximum angles of the quadrateral are at $v_{1}$ and $v_{2}$ then the locus of the fourth point $x$ giving the neutral case in the quadrilateral is along the line $v_{1} x$. In fact, we find that the segment of line $v_{1} x$ from $X_{1}=\left(r_{1}, B\right)$ to $X_{2}=\left(r_{2}, B\right)$ forms a part of the locus of $x$ where

$$
\begin{aligned}
& r_{1} v_{1} X_{1}=-l_{2} \frac{\sin (3 B+C)}{\sin B}, \\
& r_{2}=v_{1} X_{2}=-1_{2} \frac{\sin (B-C)}{\sin 3 B} .
\end{aligned}
$$

Further we observe that the neutral case for the quadrilateral $v_{1} v_{2} v_{3} x$ occurs also when the equal and maximum angles are at $v_{2}$ and $v_{3}$. The locus of such a point $x$ is the line segment from $X_{2}=\left(r_{2}, \theta_{2}\right)$ to $X_{3}=\left(r_{3}, \theta_{3}\right)$, where

$$
\begin{array}{ll}
r_{2}=-1_{2} \frac{\sin (B-C)}{\sin 3 B}, & \theta_{2}=B ; \\
r_{3}=1_{2} \frac{\sin (B-C)}{\sin B}, & \theta_{3}=360^{\circ}-3 B .
\end{array}
$$

We find that the two line segments meet at the point $X_{2}$. Therefore the locus of $x$ is the continuous broken line from $X_{1}$ to $X_{3}$.

Thus we have proved the following: 
Proposition 4.1. Given three points $v_{1}, v_{2}, v_{3}$ with $\angle v_{1} v_{2} v_{3}=B$ such that $90^{\circ}<B<120^{\circ}$, if one of the adjacent, maximum and equal angles for the convex quadrilateral $v_{1} v_{2} v_{3} x$ is at $v_{2}$ then the locus of the fourth vertex $x$ for which the neutral case occurs in the quadrilateral, consists of two line segments along $v_{1} X$ and $v_{3} X$ which meet at a point $X$, and are given by (4.1) and (4.2) respectively.

Now we are set to discuss the neutral set for points $v_{1}, v_{2}, v_{3}$ so located that $90^{\circ}<\angle B<120^{\circ}$.

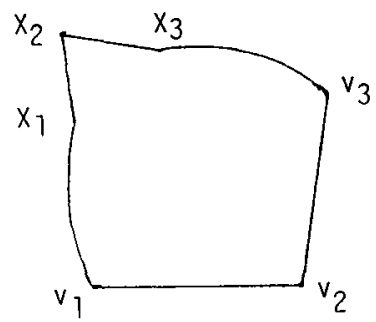

Figure 4.2

To start with the process of obtaining the neutral set in this situation, let us first suppose that $x_{1}=(r, \theta)$ is a point such that $\angle v_{2} v_{1} x_{1}$ and $\angle v_{1} x_{1} v_{3}$ are the maximum, equal and adjacent angles so that the neutral case occurs in the convex quadrilateral $v_{1} v_{2} v_{3} x_{1}$. By considering the sine formula in $\triangle v_{1} v_{2} v_{3}$, we get (3.1) as the relation between $r$ and $\theta$. Thus when $\theta$ decreases from $\theta=90^{\circ}+A / 2$ to $\theta=B$ the locus of a point $x_{1}$ is the curve $v_{1} X_{1}$ whose equation is given by (3.1).

When $\theta$ approaches value $B$, we have $\angle v_{3} v_{2} v_{1}=\angle v_{2} v_{1} x_{1}=\angle v_{1} x_{1} v_{3}$ in the quadrilateral and now we can have the maximum, equal and adjacent angles at $v_{2}$ and $v_{1}$. As discussed in Proposition 4.1 we can have a point $x_{2}$ such that $\angle x_{2} v_{1} v_{2}$ and $\angle v_{1} v_{2} v_{3}$ are the equal and maximum angles of the convex quadrilateral $v_{1} v_{2} v_{3} x_{2}$. Thus the locus of point $x_{2}$ is the line segment $X_{1} X_{2}$ given by (4.1). Further, we observe that when $x_{2}$ reaches $X_{2}, \angle x_{2} v_{3} v_{2}=B$, and now we can have the neutral case with a point $x_{3}$ such that $\angle x_{3} v_{3} v_{2}=\angle v_{3} v_{2} v_{1}$. Thus the locus of the neutral point $x_{3}$ is the line segment $X_{2} X_{3}$ given by (4.2).

At point $X_{3}$ we have $\angle v_{1} X_{3} v_{3}=\angle X_{3} v_{3} v_{2}=\angle v_{3} v_{2} v_{1}=B$ and the vectorial angle for $X_{3}$ is $360^{\circ}-3 B$. If we decrease $\theta$ still further we can have points $x_{4}$ such that $\angle v_{1} x_{4} v_{3}=\angle x_{4} v_{3} v_{2}$, and they are the maximum angles in the convex quadrilateral $v_{1} v_{2} v_{3} x_{4}$. Therefore from $\Delta v_{1} x_{4} v_{3}$, we have

$$
v_{1} x_{4}=r=1_{2} \frac{\sin (\alpha-C)}{\sin \alpha}, \quad \text { where } \alpha=\angle v_{1} x_{4} v_{3}
$$


Hence the locus of the point $x_{4}$ is the curve $X_{3} v_{3}$ whose equation is given by (3.3).

We have thus proved the following:

THEOREM 4.1. Given three points $v_{1}, v_{2}$ and $v_{3}$ with $90^{\circ}<\angle v_{1} v_{2} v_{3}<120^{\circ}$, the locus of the neutral point with respect to the min-max-angle criterion, consists of a continuous piecewise curve from $v_{1}$ to $v_{3}$ whose four pieces are given by equations (3.1), (4.1), (4.2) and (3.3) respectively.

Next we consider the case when $120^{\circ} \leqslant B<180^{\circ}$.

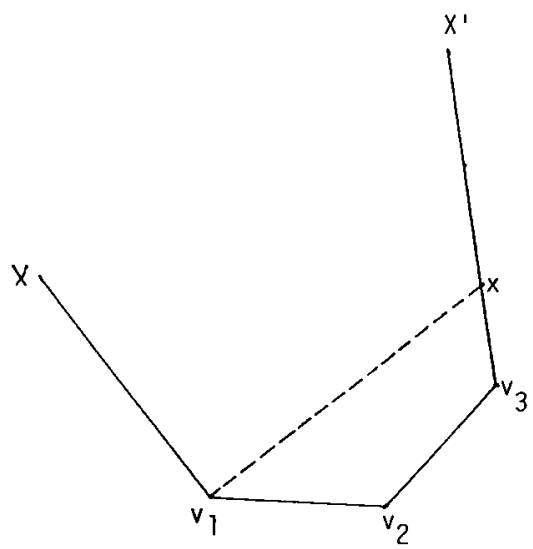

Figure 4.3

In view of Lemma 2.1, a little consideration shows that in this case, to have a neutral case in the convex quadrilateral $v_{1} v_{2} v_{2} x$ we must choose $\angle v_{1} v_{2} v_{3}$ as one of the maximum, adjacent and equal angles of the quadrilateral. Therefore, the only possible pairs of adjacent, equal and maximum angles are either $\angle v_{1} v_{2} v_{3}$ and $\angle v_{2} v_{1} x$, or the pair $\angle v_{1} v_{2} v_{3}$ and $\angle v_{2} v_{3} x$. Accordingly the locus of the neutral point is the whole line $v_{1} X$ where $\angle X v_{1} v_{2}=\angle v_{1} v_{2} v_{3}$ or the whole line $v_{3} X^{\prime}$ where $\angle v_{2} v_{3} X^{\prime}=\angle v_{1} v_{2} v_{3}$.

Clearly these two parts of the locus of point $x$ do not meet to form a closed curve.

This proves the following:

Theorem 4.2. Given three points $v_{1}, v_{2}, v_{3}$ in that order, with $\angle v_{1} v_{2} v_{3} \geqslant$ $120^{\circ}$, the locus of the fourth point $x$ such that the neutral case with respect to the minmax angle criterion occurs in the convex quadrilateral $v_{1} v_{2} v_{3} x$, consists of two infinite lines $v_{1} X$ and $v_{3} X^{\prime}$, originating from $v_{1}$ and $v_{3}$, such that $\angle X v_{1} v_{2}=\angle v_{1} v_{2} v_{3}=$ $\angle v_{2} v_{3} X^{\prime}$.

Thus given any three non-collinear points $v_{1}, v_{2}, v_{3}$, the locus of a fourth point $x$ 
such that the neutral case occurs in the quadrilateral $v_{1} v_{2} v_{3} x$, is completely determined by Theorems $3.1,4.1$ and 4.2 .

\section{ObSERVATIONS}

5.1. The neutral sets as determined by Theorems 3.1 and 4.1 are closed curves. Thus, given three points in a plane, if no angle in the triangle is larger than $120^{\circ}$, the neutral sets for them will be closed curves.

5.2. Given three points $v_{1}, v_{2}, v_{3}$, in that order such that $\angle v_{1} v_{2} v_{3}<120^{\circ}$, so that the neutral set is a closed curve and if the fourth point $v_{4}$ is outside this closed curve, the triangulation with $v_{1} v_{3}$ as one edge would be better than that for choice $v_{2} v_{4}$ as an edge. This observation gives a procedure for obtaining a better triangulation with respect to the min-max-angle criterion.

5.3. In the case when $B \geqslant 120^{\circ}$, the neutral set is not a closed curve, but consists of two infinite lines. Then for a point $v_{4}$ lying inside this region, edge $v_{2} v_{4}$ should be preferred to have a better triangulation.

\section{A PRACTICAL ALgORIghM}

A few preliminaries are needed before we define the algorithm:

Definition 6.1: Triangle Number. Triangle numbers are given from 1 onwards. When a new triangle is formed we give it the next number.

Definition 6.2: Status of a triangle.

Alive: Every newly formed triangle is alive.

Dead: $\quad 1$. If it looses its existence,

or 2. All its sides are dead.

Definition 6.3: Status of a side.

Alive: A side of a triangle is alive by default,

Dead: 1. If it is a common edge of two triangles,

2. If it loses its existence,

3. If it is on the boundary of the convex hull containing $v_{1}, v_{2}, \ldots, v_{n}$.

REMARK. When a triangle dies all its sides also die. A side which has died may become alive in a new triangle.

Algorithm.

Step 1 Choose any three points amongst $v_{i} i=1,2, \ldots, n$, such that they form a triangle and there is no point inside or on the boundary of the triangle. 
Mark the triangle as 'alive'. Also mark the status of sides as alive or dead (according to Definition 6.3). Set 'number of triangles considered' to 0 .

Step 2 Consider the alive triangle whose number is (number of triangle considered +1 ).

If this is dead then consider the next triangle whose number is 1 more and so on until we get an alive triangle. If no alive triangle is left, go to Step 6.

Set 'number of sides considered' to 0 .

Step 3 Consider an alive side of the triangle under consideration.

If all its sides are dead go to Step 2.

Step 4 Suppose the triangle under consideration is $A B C$ and the side under consideration is $A C$.

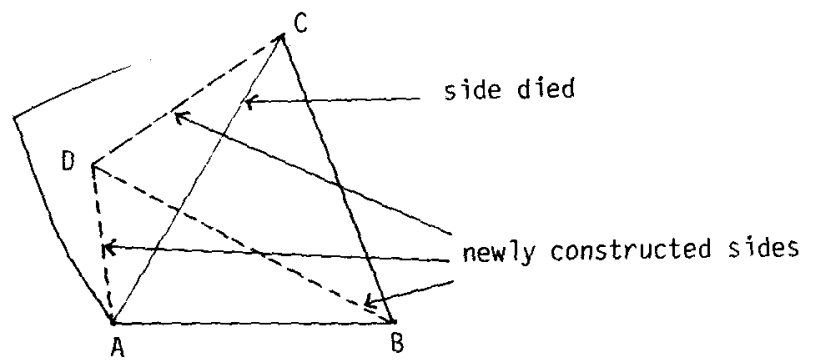

Figure 6.1

Consider the neutral set curve of $\triangle A B C$ opposite to the side $A C$. Choose a proper point $D$. (Definition of Proper point: A point $D$ is a proper point if

1. It is not on $A C$.

2. $A D, B D, B C$ do not cross any of the sides of existing triangles.

3. $\triangle A D C$ contains no point from the $v_{i}$ 's.)

If we are unable to choose $D$ inside a neutral set go to Step 5 .

If there are more than one proper points then choose any one of them (and call it $D$ ).

Form $\triangle A B D$ and give it the next number.

Form $\triangle B D C$ and give it the next number.

Mark sides $A D$ and $C D$ as alive and $A C$ as dead.

The status of $A B$ and $B C$ remain the same.

Mark $\triangle A B C$ as dead.

Go to Step 4 (again). 
Step 5 If there is no proper point inside the neutral set we consider a proper point outside the neutral set curve. Preferably the point chosen should be near $A C$. (If no such point exists, the triangulation is complete. Go to step 6). Let the point be $D$.

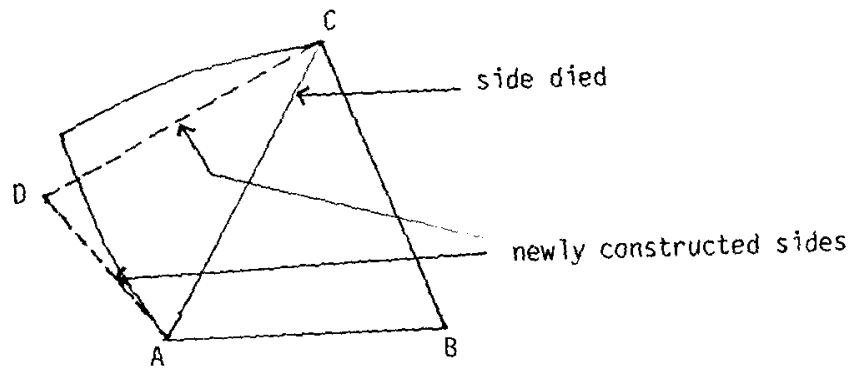

Figure 6.2

Then form the triangle $A C D$ and assign it the next triangle number. Mark $A C$ as dead in $\triangle A B C$ and $\triangle A C D$. Go to Step 3.

Step 6 The triangulation is complete.

\section{REFERENCES}

[1] Y. Correc and E. Chapius, 'Fast computation of Delaunay triangulations', Adv. Eng. Software 9 (1987), 77-83.

(2) D. Field, 'A flexible Delaunay triangulation algorithm', General Motors Research Publ. (No. GMR-5675) (1987).

[3] D. Hansford, 'The neutral case for the min-max triangulation', Comput. Aided Geom. Design 7 (1990), 431-438.

[4] C.L. Lawson, 'Generation of triangular grid with application to contour plotting', J. P.L. $299(1972)$.

[5] L.L. Schumaker, 'Triangulation methods', in Topics in multivariate approximation, (C.K. Chui, L.L. Schumaker and F.I. Utreras, Editors) (Acsdemic Press, New York, 1987), pp. 219-232.

Department of Mathernatics and Computer Science

R.D. University

Jabalpur

India 